\title{
Prevalence of sarcopenia and associated factors in older adults attending a day hospital service in Ireland
}

\author{
Kieron Connolly ${ }^{1,4} \oplus \cdot$ Conal Cunningham ${ }^{2} \odot \cdot$ Niamh Murphy $^{1}\left([) \cdot\right.$ Roman Romero-Ortuno $^{2,3}\left(\mathbb{C} \cdot\right.$ Frances Horgan $^{4} \oplus$
}

Received: 28 October 2020 / Accepted: 30 January 2021 / Published online: 13 February 2021

(c) European Geriatric Medicine Society 2021

\section{Key summary points}

Aim The aim of this study was to describe the prevalence and associated factors of sarcopenia in community-dwelling older adults attending a day hospital service in Ireland, using the latest European Working Group of Sarcopenia in Older People (EWGSOP) guidelines.

Findings The prevalence of sarcopenia in older adults attending a day hospital service in Ireland was $27-37 \%$ and varied depending on the strength test used. Sarcopenia was associated with older age, female gender, reduced BMI, reduced calf circumference, frailty, malnutrition, and reduced gait speed, irrespective of how muscle strength was assessed.

Message Further consideration on the assessment of sarcopenia needs to be considered, regarding the use of muscle strength assessment, whether to assess upper limb, lower limb, or both.

\begin{abstract}
Purpose Sarcopenia is a muscle disease that is linked to the effects of ageing, chronic diseases, physical inactivity, and poor nutrition. In Ireland, there is a lack of readily available information on sarcopenia in older adults. The aim of this study was to describe the prevalence and associated factors of sarcopenia in community-dwelling older adults attending a day hospital service in Ireland, using the European Working Group of Sarcopenia in Older People (EWGSOP) guidelines.

Methods An observational cross-sectional study was conducted, where a consecutive series of older adults attending a day hospital service were invited to participate. The measure of primary interest was the diagnosis of sarcopenia using the EWGSOP 2019 guidelines. We also collected other Comprehensive Geriatric Assessment measures including cognition, nutrition, frailty and physical activity.

Results A total of 134 participants took part in the study. The mean age was 81.7 ( $\mathrm{SD} \pm 7.1)$. Sixty-one percent $(N=82)$ were female. The prevalence of sarcopenia ranged from 27 to $37 \%$ depending on the assessment tool used to assess muscle strength. Sarcopenia was associated with older age, frailty, reduced nutritional state, poor physical performance and reduced anthropometric measures, irrespective of how muscle strength was measured. Independently associated factors differed depending on muscle strength test, except for older age.

Conclusion The prevalence of sarcopenia in the day hospital ranged from 27 to $37 \%$. The assessment tool used to assess muscle strength influenced both the prevalence and associated factors of sarcopenia, suggesting scope for further research.
\end{abstract}

Keywords Sarcopenia $\cdot$ Elderly $\cdot$ Day hospital $\cdot$ Frail $\cdot$ Muscle strength

Kieron Connolly

kieron.connolly@ucdconnect.ie

1 Physiotherapy Department, St James's Hospital, Dublin 8, Ireland

2 St James's Hospital, Mercers Institute of Successful Ageing, Dublin 8, Ireland
3 Department of Medical Gerontology, Trinity College Dublin, Dublin 2, Ireland

4 School of Physiotherapy, Royal College of Surgeons in Ireland (RCSI) University of Medicine and Health Sciences, Dublin 2, Ireland 


\section{Introduction}

Sarcopenia has been defined as a progressive and generalised skeletal muscle disorder that occurs in advanced ageing and results in reduced muscle mass, reduced muscle strength and poor muscle function [1]. Sarcopenia is associated with increased mortality, disability, risk of falls and increased cost of care during hospital admissions [1-4]. The main factors related to the development of sarcopenia include the effects of ageing, poor nutrition, physical inactivity, and the presence of chronic diseases [5]. However, associations may be influenced by different sarcopenia operationalisations.

Several groups have published definitions and diagnostic procedures for sarcopenia, including the European Working Group of Sarcopenia for Older People (EWGSOP). In 2019, EWGSOP updated their definition of sarcopenia (EWGSOP2) [6], and the change in definition placed a greater emphasis on the importance of muscle strength due to its stronger relationship with adverse outcomes. For example, Dos Santos et al. [7] showed that loss of muscle strength had an increased risk of losing physical independence compared to a loss of muscle mass in community-dwelling older adults. EWGSOP2 are the only criteria to define sarcopenia as reduced muscle strength and mass, while physical performance is used to define the severity of sarcopenia [6].

Ireland has experienced an increase in the number of older adults (aged $>65$ years) in the last decade; and the Central Statistics Office (CSO) has predicted that, by 2030, one in five Irish people will be over the age of 65 [8]. With an increasing ageing population, the prevalence of sarcopenia is likely to increase, leading to further demands on healthcare systems. Currently, the prevalence of sarcopenia in Irish studies ranges from 30 to $41 \%$ but these studies have focused on specific population groups including those with advanced oesophageal cancer, limiting our understanding of sarcopenia prevalence in older adults in Ireland [9]. In community-dwelling older adults, the prevalence of sarcopenia is widely debated, ranging from 0 to $50 \%$ [10].

The early identification of sarcopenia is important for preventing its adverse outcomes in older adults [5]. One essential component of the model of care of older adults in Ireland is the day hospital service [11]. This service provides rapid access medical and nursing care as well as interdisciplinary and ambulatory care services [12]. Those attending a day hospital receive medical care and multi-disciplinary intervention, due to a change or decline in their health or function. To date, there is no information regarding the prevalence of sarcopenia in community-dwelling older adults attending a day hospital service in Ireland. Identifying the prevalence of sarcopenia in this group would enable more comprehensive and timely management of sarcopenia.
Aim

The aim of this study was to establish the prevalence and associated factors of sarcopenia in community-dwelling older adults attending a day hospital service in Ireland.

\section{Methods}

A consecutive series of older adults attending the Robert Mayne Day Hospital (RMDH) in St James's Hospital (SJH) Dublin, Ireland were invited to take part in the study between October 2019 and March 2020. Ethical approval was granted by the Hospital Research Ethics Committee (2019-10-39). The study was designed and reported in accordance with the 'Strengthening the reporting of Observational Studies in Epidemiology' (STROBE) guidelines. The clinical nurse managers (CNMs), RMDH consultants and medical registrars acted as gatekeepers for the study. The gatekeepers screened patients in accordance with the study's inclusion and exclusion criteria to determine eligibility for participation. All suitable patients were provided with a study patient information leaflet (PIL). Older adults ( $>65$ years of age), living in the community, who could sit to stand independently (with or without an aid/appliance), provide informed consent, understand simple instructions to allow the completion of assessments and could manipulate a wrist strap physical accelerometer were invited to participate in the study. Individuals who were medically unstable had a contraindication to Bio-Impedance Analysis (BIA) (e.g. pacemaker, implantable cardioverter device, amputation), who had a Clinical Frailty Scale (CFS) of 9 or greater or life expectancy $<3$ months or who were currently or previously undergoing cancer treatment (chemotherapy or radiotherapy) in the last year were excluded. The latter exclusion criterion was implemented to limit the effect of cancer-related cachexia on sarcopenia prevalence.

All potential participants were given at least one week to consider participation in the study before providing informed consent. All assessments (which lasted between 30-60 min) took place in the physiotherapy gym of the RMDH by the principal researcher to avoid inter-rater bias. Demographic information was collected using the participants' medical chart and electronically using the hospital electronic patient record system (EPR). Demographic information included participants' age, gender, social deprivation index, past medical history, comorbidity status ( $>6$ conditions), polypharmacy ( $>6$ medications), living status, presence of home care package, use of meals on wheels service, falls in the last year, hospitalisation in the last year and prescription of vitamin D supplements. The Modified Cumulative Illness 
Rating Scale (m-CIRS) [13] was recorded and the scoring system developed by Salvi et al. [14] was implemented. The m-CIRS was used to assess the severity of comorbidities in this study. The m-CIRS grades comorbidities among 13 different organ systems and grades each condition from 0 (no problem) to 4 (severely incapacitating or life-threatening conditions) [14]. A higher m-CIRS score is indicative of higher disease burden/severity.

The primary research measure in our study was the presence of sarcopenia and category of sarcopenia using the EWGSOP2 criteria [6]. In the EWGSOP2 criteria, sarcopenia was defined as the presence of low muscle strength and low muscle mass. Probable sarcopenia was defined as reduced muscle strength only (normal muscle mass). Severe sarcopenia was defined as the presence of sarcopenia in combination with reduced physical performance.

Muscle strength was assessed using both handheld grip strength (reduced grip strength $=<27 \mathrm{~kg}$ for men and $<16 \mathrm{~kg}$ for women) and the Five Times Sit to Stand Test (5-STS) (reduced 5STS time $=<15 \mathrm{~s}$ ). Hand-held grip strength assessment procedure was standardised using Roberts et al.'s [15] recommendations and the strongest measurement from 6 tests $(\times 3$ right and $\times 3$ left $)$ was used. The 5-STS test assessment was standardised using the Short Physical Performance Battery (SPPB) instructions [16].

Muscle mass was assessed using Appendicular Skeletal Mass (ASM) adjusted for height using raw data from BIA (Bodystat Quadscan 4000 Touch) and validated predictive equations [17, 18]. The Sergi et al. [17] equation predicts ASM from $-3.964+\left(\right.$ height $[\mathrm{cm}]^{2} /$ resistance $\times 0.227)+(0.095 \times$ body weight $[\mathrm{kg}])+(1.384 \times$ sex $[\operatorname{men}=1$, women $=0])+(0.064 \times$ reactance $)$. The Scafoglieri et al. [18] equation predicts ASM from $4.957+$ (height $[\mathrm{cm}]^{2} /$ resistance $\left.\times 0.196\right)+(0.060 \times$ body weight $[\mathrm{kg}])$ $-(2.554 \times \operatorname{sex}[\mathrm{men}=0$, women $=1])$. The cut-offs used to define low muscle mass were set at ASM $<7 \mathrm{~kg} / \mathrm{m}^{2}$ for men and $<6 \mathrm{~kg} / \mathrm{m}^{2}$ for women. All participants were positioned supine on a plinth for the assessment and 4 electrodes were placed on each participant's right side $(\times 2$ dorsal aspect of the right hand and $\times 2$ on the dorsal aspect of right foot). Fluid intake and bladder voiding were not controlled for in this study.

Physical performance was measured using the SPPB $(<8 / 12)$ and/or the Timed Up and Go Test (TUG) $(>20 \mathrm{~s})$ using instructions from Herman et al. [19]; the cut-offs were as suggested in the EWGSOP2 criteria [6].

Secondary measures included blood markers, anthropometric measures, risk of sarcopenia, cognition, frailty, physical activity, and nutritional status. The most recent blood markers were used for haemoglobin $(\mathrm{Hb})$, vitamin $\mathrm{D}$ (25-OH-Vitamin D) and C-reactive protein (CRP). All values for blood markers were obtained from the EPR system or the medical chart. Anthropometric measures included height, weight, body mass index (BMI) and calf circumference.

An individuals' risk of sarcopenia was assessed using the SARC-F questionnaire (Strength, Assistance with walking, Rise from a chair, Climb stairs and Falls), where a score of $>4$ was indicative of risk. Nutritional status was assessed using the modified Mini Nutritional Assessment- Short Form (MNA-SF). An individual is classified as malnourished with scores of $<7$, risk of malnutrition if $8-11$ and well-nourished/normal if scores $>12$ [20].

Cognition was assessed using the Quick Mild Cognitive Impairment Screen (QMCI). The components of the QMCI include orientation (/10), word registration (/5), clock drawing (/15), delayed recall (/20), verbal fluency (/20) and logical memory (/30) [21]. These areas are scored differently, with a total QMCI score of 100 , with $<62 / 100$ being predictive of mild cognitive impairment (MCI) or dementia [21].

Frailty was assessed using the CFS and the SHARE Frailty Instrument (SFI). The SFI is based on a modified phenotypic approach and includes two web-based frailty calculators for each gender [22]. Both calculators are freely accessible on http://www.biomedcentral.com/ 14712318/10/57/additional. Each participant's SFI scores were inputted into the calculators to determine frailty status [22].

Physical activity levels were measured using the International Physical Activity Questionnaire for Elderly (IPAQ-E). The IPAQ-E was scored using the IPAQ scoring protocol [23] (https://www.sites.google.com/site/theipaq/home). When scoring the questionnaire, metabolic equivalents (METS) per week were calculated for walking and moderate and vigorous physical activity (PA), while time spent sitting per week (min) was also documented. For walking, MET 3.3 was used; and for moderate and vigorous activity, METS 4.0 and 8.0 were used as per scoring protocol [23]. An individual was classified as having low PA levels if their weekly METS fell below 600 a week, moderate PA levels were classified as METS of 601-2999 a week, while high PA levels were classified as $>3000$ METS a week [23].

\section{Statistical analysis}

Sample size was based upon the attendance record of the RMDH over 12 months (approx. 5030 patients), and Conroy's [24] sample size guide for prevalence studies, accounting for 5\% loss to follow up and estimating a prevalence of approximately $50 \%$. The sample size estimate for the study was 180 participants. Microsoft Excel and SPSS v26 (IBM Corp) were used for data analysis. Pearson's $R$ (normally distributed variables) and Spearman's Rho correlation coefficient (non-parametric) tests were used to assess the strength of correlation between variables. A correlation coefficient $\geq 0.6$ was considered strong, between 0.3 and 0.59 
moderate, and $<0.3$ mild. Bivariate analyses were conducted using four different tests, depending on the type of variable and the distribution of the said variable. For normally distributed, continuous variables, independent $T$ tests were used to compare sarcopenia groups. For non-parametric continuous variables, the Mann-Whitney $U$ test was used. Pearson's Chi-square test was used to determine associations between dichotomous variables and sarcopenia presence; $\mathrm{Phi}$ value was determined using SPSS and was used to assess the effect size of the relationship. The linear-by-linear association (trend) test was used to determine associations between sarcopenia presence and ordinal scales (3-6 categories); the Cramer value was determined using SPSS and was used to assess the effect size of the relationship. Multivariate logistic regression analysis, using the Peduzzi rule, was used to determine independent predictors of sarcopenia [25]. To be determined statistically significant, the $p$ value was set at $<0.05$.

\section{Results}

\section{Profile of study participants}

A total of 134 participants were recruited for the study (Fig. 1). They had a mean age of 81.7 (SD 7.1), with a range from 65 to 98 . Sixty-one percent $(N=82)$ were women. A summary of their demographic information is shown in Table 1 . The median $\mathrm{m}$-CIRS was $9 / 56$ [interquartile range (IQR) 5]. The median BMI was 25.7 (IQR 7), 31\% $(N=42)$ were classified as overweight and nearly one quarter had obesity $(23 \%, N=32)$.

Just over one-third of participants were prescribed a vitamin D supplement $(N=46,34 \%)$, with just over half of the participants having normal serum vitamin D levels $(N=76$, $57 \%)$. The remaining $29 \%(N=39)$ had low vitamin D levels, $15 \%(n=20)$ had vitamin D insufficiency and $14 \%(N=19)$ had vitamin D deficiency. Vitamin D values were missing for $16 \%(N=19)$. Using the World Health Organisation (WHO) anaemia definition (serum haemoglobin level $<13 \mathrm{~g} / \mathrm{dL}$ in men and $<12 \mathrm{~g} / \mathrm{dL}$ in women), the prevalence of anaemia was $40 \%(N=54)$, with $54 \%(n=28)$ of men and $32 \%$ $(N=26)$ of women being defined as anaemic.

Only $46 \%(N=61)$ of participants were regarded as 'at risk' for developing sarcopenia $(>4)$ using the SARC-F. The median QMCI score was 50.8 (IQR 22.5), indicating MCI $(<62 / 100)$. The prevalence of MCI was $27 \%(N=36)$, with $34 \%(N=46)$ of participants categorised as having normal cognition. The median CFS score was 4 (IQR 2), ranging from $1-7$. For the SFI $(N=133)$, the median score was 2.43 (IQR 2.34). Using the SFI, 50\% $(N=67)$ of participants were classified as frail, while $29 \%(N=38)$ were regarded as pre-frail, with the remaining $21 \%(N=28)$ being non-frail. Forty percent of participants $(N=53)$ had a normal nutritional state, while $46 \%(N=61)$ were identified as 'At risk' of malnutrition with $14 \%(N=19)$ highlighted as being 'Malnourished'. Using the IPAQ-E, 51\% $(N=68)$ were classified as being in the low physical activity (PA) category, with 35\% $(N=47)$ being in the moderate PA category and only $14 \%(N=19)$ in the vigorous PA category. The median time for sitting of $360 \mathrm{~min}$ (IQR 197.5) per day equated to $6 \mathrm{~h}$ per day.

Grip strength ranged from 2 to $48 \mathrm{~kg}$ with the median ranging from 16 to $19 \mathrm{~kg}$ (IQR 10-12) across the six trials. The gait speed time ( $3 \mathrm{~m}$ distance measured as a part SPPB) ranged from 1.6 to $12.5 \mathrm{~s}$ (median $4.1 \mathrm{~s} \mathrm{IQR} 2.2 \mathrm{~s}$ ). The best TUG time was a median $13.9 \mathrm{~s}$ (IQR $10.9 \mathrm{~s}$ ). Only 73 participants could complete the 5-STS test, due to the inability of 59 participants to perform one STS with upper limb support. As per the SPPB scoring criteria, an individual must be able to complete one STS without upper limb support before being able to start the 5-STS test. From the 5-STS scores, only $27 \%(N=36)$ participants in the study had normal lower limb (LL) strength as per the EWGSOP2 criteria $(5-\mathrm{STS}<15 \mathrm{~s})$. Using the SPPB, $59 \%(N=79)$ of participants had poor physical performance $(\mathrm{SPPB}<8)$, in comparison to the TUG, which identified only $27 \%(N=36)$ as having poor physical performance.

\section{Sarcopenia prevalence and associated Factors}

When using grip strength to categorise muscle strength, 27\% $(N=35)$ of participants were sarcopenic and $95 \%(N=28)$ of those who were sarcopenic had severe sarcopenia. In comparison, when using LL strength (5-STS) to categorise muscle strength, $37 \%(N=49)$ were sarcopenic and of those sarcopenic, $84 \%(N=41)$ had severe sarcopenia. A breakdown of the two criteria can be seen in Table 2. Sarcopenia classified using grip strength is referred to as sarcopenia (grip) while sarcopenia classified using LL strength (5-STS) is referred to as sarcopenia (LL). Sarcopenia (grip and LL) refers to both sarcopenia (grip) and sarcopenia (LL).

When sarcopenia was determined as present or absent (sarcopenia vs normal), the two criteria [sarcopenia (grip) and sarcopenia (LL)] agreed strongly $(\kappa=0.62)$. However, the strength of the agreement decreased to a moderate level, using all four categories of the EWGSOP2 criteria (normal, probable sarcopenia, sarcopenia, and severe sarcopenia) $(\kappa=0.53)$. There was a mild-moderate correlation between the SARC-F findings and the classification of participants using all categories of the EWGSOP2 criteria (Spearman Rho: $0.2-0.3 ; p<0.05)$. However, there was no correlation between the SARC-F and the classification of participants as sarcopenic or normal ( $p=0.152,0.197)$. When diagnosing sarcopenia, two calculations of ASM were used, the Scaf and Sergi equations, to define low muscle mass. Using the Scaf 
Fig. 1 Flow of participants in the study $(N=134)$. *The number of attendances to the RMDH from October 2019 to March 2020 was 1813 , this number was determined by calculating the number of patients booked in for the day hospital each day. The study was conducted in a busy day hospital clinical environment where the gatekeepers who were members of the clinical team were also asked to screen for eligible study participants. The specific reasons for exclusion were not recorded but would have included age, excessive frailty, and severe cognitive impairment

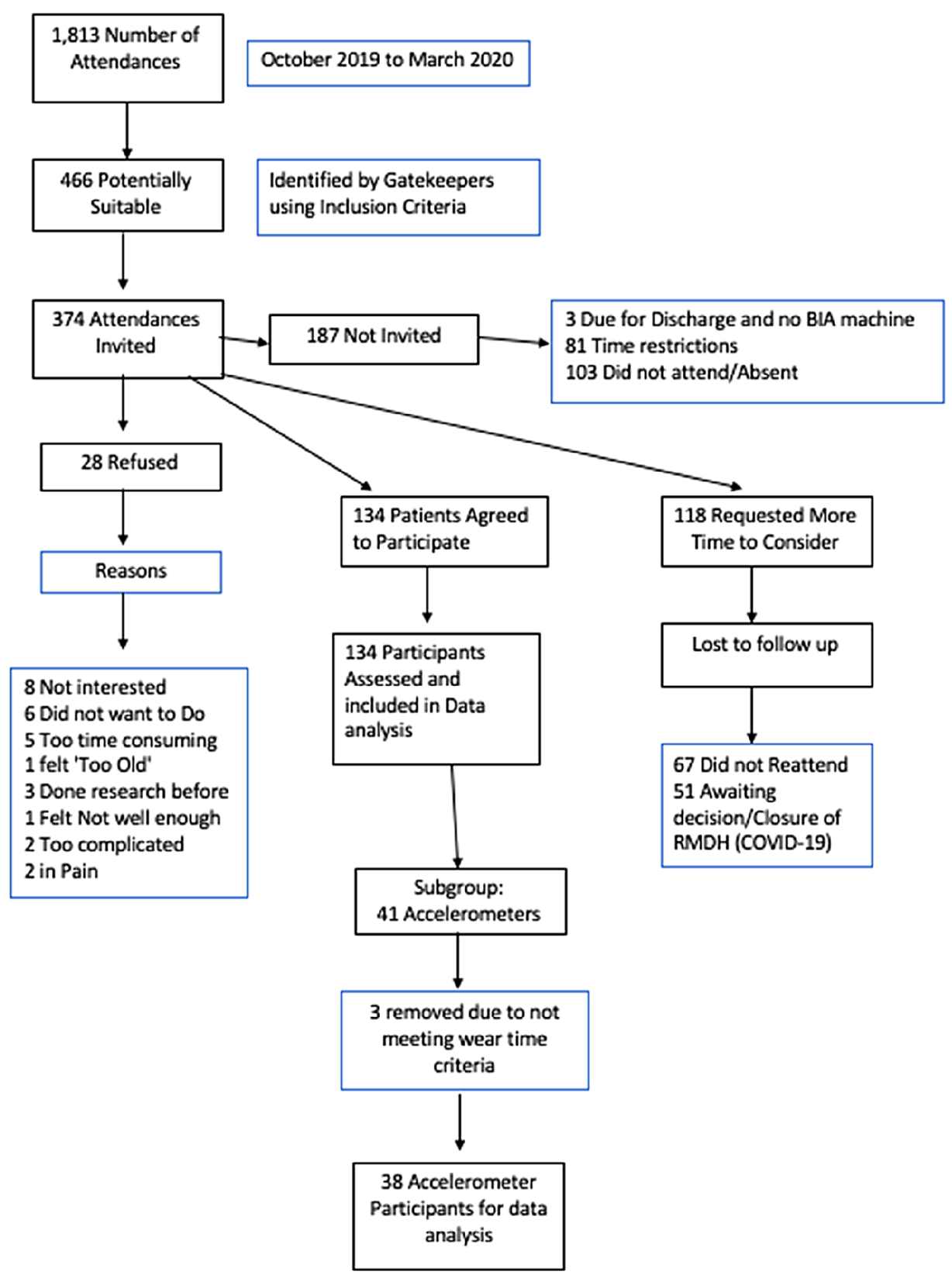

equation, $47 \%(N=62)$ of participants had low muscle mass using BIA. Using the Sergi equation, $48 \%(N=63)$ of participants had low muscle mass using BIA. An agreement on low muscle mass classification, between the two equations, was established at $83 \%(N=111)$ of cases. The two equations were highly correlated (Pearson correlation $=0.911$, Spearman correlation $=0.909, p=0.001$ ).

The common associated factors of sarcopenia (grip) and sarcopenia (LL) can be seen in Table 3. Sarcopenia (grip) and Sarcopenia (LL) were both associated with older age and reduced $\mathrm{Hb}$ levels. There were significant differences between the sarcopenia (grip) group and non-sarcopenic group in relation to age ( $T$ test: -4.997 , $p<0.001)$ and Hb levels $(Z=-2.914, p=0.04)$. Sarcopenia (LL) was also associated with older age ( $T$ test: $-4.356, p<0.001)$ and reduced Hb levels $(Z=-2.601$, $\mathrm{p}=0.009$ ). Sarcopenia (grip and LL) was mildly associated with female gender (Phi 0.23-0.25) (Pearson Chi square $=6.9-8.2, p=0.004-0.008)$ and a history of osteoporosis or osteopenia (Chi square 6.7-6.8, $p=0.009$, Phi 0.23 ). Sarcopenia (grip and LL) was associated with a reduction in various anthropometric measures including height, weight, body mass index (BMI) and calf circumference $(\mathrm{CC})(p<0.05)$. Sarcopenia (Grip and LL) was 
Table 1 Description of the study participants $(N=134)$

\begin{tabular}{ll}
\hline Variable & $N(\%)$ \\
\hline Age years, mean (SD) & \\
Range 65-98 & $81.7(7.1)$ \\
Gender & \\
Men & $52(39)$ \\
Women & $82(61)$ \\
Living status & \\
Lived alone & $60(45)$ \\
Lived with someone & $74(55)$ \\
Supports & \\
HCP & $20(15)$ \\
MOW & $9(6.7)$ \\
Reason for referral to RMDH & \\
Outpatients & $79(59)$ \\
Recent inpatient stay & $42(31)$ \\
GP referral & $3(2)$ \\
Recent attendance at ED & $10(8)$ \\
Social deprivation index living area \\
'Marginally below average' & \\
'Marginally above average'' & \\
'Disadvantaged/very disadvantaged' & $54(40)$ \\
'Affluent/very affluent' & $40(30)$ \\
Comorbidities >6 & $14(10)$ \\
Comorbidity details & $26(20)$ \\
Rheumatology & $82(61)$ \\
Lung disease & \\
Cancer history & \\
Osteoporosis/osteopenia & \\
Chronic kidney disease & $41(9)$ \\
Polypharmacy & $32(24)$ \\
>6 medications & $41(31)$ \\
\hline
\end{tabular}

$H C P$ home care package, $M O W$ meals on wheels

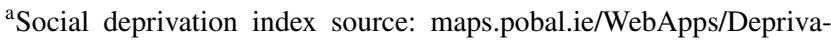
tionIndices/index.html

also associated with reduced LL strength/5-STS time, reduced gait speed, reduced grip strength and poor physical performance (as measured by the SPPB $<8)(p<0.05)$. Sarcopenia (grip and LL) was also associated with the reduced nutritional state using both the Mann-Whitney $U$ test and linear by linear association test $(p<0.05)$. Frailty, as measured using the SFI, was associated with sarcopenia (grip and LL) using the linear by linear association test $(p<0.05$; Cramer $=0.21-0.26)$ and Chi-square test $(p<0.05$; Phi 0.18-0.19), while an increased CFS was associated with sarcopenia (grip and LL) $(p<0.05)$. Only sarcopenia (grip) was associated with a history of chronic kidney disease (CKD) (Chi square: $4.86 p=0.027$ Phi 0.19) and hospitalisation in the last 12 months (Chi square: $4.37 p=0.037$, Phi 0.18 ). Only sarcopenia (LL) was associated with time to complete TUG $(Z=-2.667$, $p=0.008)$, total SPPB score $(Z=-2.7, p=0.007)$, falls in last year (Chi square: $6.75, p=0.009$, Phi $=0.23$ ) and cognition (normal vs impaired) (Chi square: 4.92 $p=0.027$, Phi 0.19).

\section{Factors independently associated with sarcopenia}

Post bivariate analysis, a total of 33 individual variables were found to be significantly associated with sarcopenia (grip) $(p<0.05)$ while 37 individual variables were associated with sarcopenia (LL) $(p<0.05)$. Some of these variables overlapped between binary and ordinal outcomes of the same questionnaires/scales (i.e. CFS). Accounting for this, there were 16 unique variables associated with sarcopenia (grip) and 19 unique variables associated with sarcopenia (LL). Due to the differences between sarcopenia (grip) and sarcopenia (LL), regression models were developed for each, separately.

For both regression models, age and gender were included. Age was not strongly correlated with any variable, therefore its inclusion in the equation would not have inflated any significant findings. To prevent multi-collinearity, body mass measures such as BMI, CC and height were not inputted due to their strong correlations with all muscle mass variables $(R \geq 0.6)$. Grip strength was not strongly correlated with any physical performance measure or LL strength, which enabled the inclusion of other strength and physical performance measures to sarcopenia (grip) regression models. LL strength (5-STS time) was strongly associated $(R>0.06)$ with all other physical performance measures except grip strength $(R<0.6)$, so only grip strength was included in the regression models for sarcopenia (LL).

For sarcopenia (grip), the regression model included age, gender, vitamin D supplement (yes or no), SPPB score, modified MNA-SF total, CFS, history of CKD (yes or no), and a history of osteopenia or osteoporosis (yes or no) (Table 4). The regression model identified older age $(p=0.001)$, female gender $(p=0.04)$ and a history of CKD $(p=0.034)$ as independently associated with sarcopenia (grip). The regression model performed moderately well with an area under the curve (AUC) of 0.85 (95\% confidence interval 0.79-0.92) and a Nagelkerke $R$ square $\left(R^{2}\right)$ of 0.42 with a correct classification as sarcopenic or not sarcopenic in $80 \%$ of cases.

For sarcopenia (LL), the regression model included age, gender, best grip strength measurement, modified MNA-SF classification (normal or reduced), CFS, QMCI classification (normal or impaired) and $\mathrm{Hb}$ levels (Table 5). The regression model identified older age $(p=0.004)$ and reduced nutritional state $(p=0.024)$ as independent predictors of sarcopenia (LL). The regression model performed moderately well with an AUC of 0.83 (95\% confidence interval 0.76-0.91) and a Nagelkerke $R$ square $\left(R^{2}\right)$ of 0.35 with a correct classification as sarcopenic or not sarcopenic in $76 \%$ of cases. 
Table 2 Prevalence of sarcopenia (grip) and sarcopenia (LL)

\begin{tabular}{|c|c|c|c|c|c|c|}
\hline \multirow[t]{2}{*}{ Sarcopenia categories } & & & \multicolumn{4}{|c|}{ Prevalence $\%(N)$} \\
\hline & & & \multicolumn{3}{|c|}{ Sarcopenia (grip) } & Sarcopenia (LL) \\
\hline Normal & & & \multicolumn{2}{|c|}{$51 \%(68)$} & & $29 \%(38)$ \\
\hline Probable sarcopenia & & & \multicolumn{2}{|c|}{$23 \%(30)$} & & $33 \%(44)$ \\
\hline Sarcopenia & & & \multicolumn{2}{|c|}{$27 \%(35)$} & & $37 \%(49)$ \\
\hline Severe sarcopenia $^{\mathrm{a}}$ & & & \multicolumn{2}{|c|}{$21 \%(28)^{\mathrm{a}}$} & & $31 \%(41)^{\mathrm{a}}$ \\
\hline Total & & & \multicolumn{2}{|c|}{133} & & 131 \\
\hline \multirow[t]{3}{*}{ Sarcopenia categories } & \multicolumn{6}{|c|}{ Prevalence \% $(N)$ and gender breakdown } \\
\hline & \multicolumn{2}{|c|}{ Sarcopenia (grip) } & \multirow[t]{2}{*}{$p$} & \multicolumn{2}{|c|}{ Sarcopenia (LL) } & $p$ \\
\hline & Men & Women & & Men & Women & \\
\hline Normal & $55 \%(28)$ & $49 \%(40)$ & 0.1 & $28 \%(14)$ & $30 \%(24)$ & 0.1 \\
\hline Probable Sarcopenia & $31 \%(16)$ & $17 \%(14)$ & & $50 \%(25)$ & $24 \%(19)$ & \\
\hline Sarcopenia & $14 \%(7)$ & $34 \%(28)$ & & $22 \%(11)$ & $47 \%(38)$ & \\
\hline \multirow[t]{3}{*}{ Sarcopenia (binary) } & \multicolumn{6}{|c|}{ Prevalence $\%(N)$ and gender breakdown } \\
\hline & \multicolumn{2}{|c|}{ Sarcopenia (grip) } & \multirow[t]{2}{*}{$p$} & \multicolumn{2}{|c|}{ Sarcopenia (LL) } & \multirow[t]{2}{*}{$p$} \\
\hline & Men & Women & & Men & Women & \\
\hline Normal & $86 \%(44)$ & $65 \%(53)$ & $0.008^{*}$ & $78 \%$ (39) & $53 \%(43)$ & $0.004 *$ \\
\hline Sarcopenia & $14 \%(7)$ & $35 \%(28)$ & & $22 \%(11)$ & $47 \%(38)$ & \\
\hline
\end{tabular}

$* p<0.05$

${ }^{\text {a }}$ Severe sarcopenia is the percentage of those who have sarcopenia, not of total number of participants

\section{Discussion}

The prevalence of sarcopenia in community-dwelling older adults attending a day hospital service in this study was $27-37 \%$ using the EWGSOP2 criteria. Of those who were sarcopenic, high proportions were classified as having severe sarcopenia [95\% of those in sarcopenia (grip) and $84 \%$ of those in sarcopenia (LL)]. Sarcopenia (grip and LL) was associated with older age, female gender, a history of osteoporosis/osteopenia, reduced $\mathrm{Hb}$ levels, reduced muscle strength, reduced muscle mass, poor physical performance $(\mathrm{SPPB}<8)$, reduced gait speed, reduced nutritional state, frailty and reduced anthropometric measures (including calf circumference and BMI). Sarcopenia (grip and LL) was independently associated with older age, while sarcopenia (grip) was independently associated with being female and having a history of CKD. In comparison, sarcopenia (LL) was independently associated with the decreased nutritional state (modified MNA-SF).

The prevalence found in our study was similar to that of the Newcastle 85 + study [26], where the prevalence of sarcopenia using the EWGSOP1 criteria and grip strength was found to be $21 \%(N=149)$. In Spain, in a geriatric outpatient setting, the prevalence of sarcopenia was established at $19 \%$ using EWGSOP1 [27]; in that study, the median age was 83.2 , which is similar to our day hospital group, with similar proportions of men and women. In Reiss et al. [28], with a sample of 144 older inpatients, the EWGSOP1 criteria yielded a prevalence rate of $28 \%$ compared to $18 \%$ (EWGSOP2). In our study, the LL strength assessment identified more individuals as sarcopenic compared to grip strength. Sobestiansky et al. [29] established a similar prevalence rate to our findings when using the EWGSOP1 (21\%) and EWGSOP2 (20\%) in 287 healthy community-dwelling older men 85-89. The participants were more closely related to the day hospital population in our study as $34 \%$ had impaired LL strength, average gait speed was $1.36 \mathrm{~m} / \mathrm{s}$ and mean grip strength was $30 \mathrm{~kg}$ [29], although men were slightly older (mean age 86.6). The prevalence was higher in our study and could be attributed to the muscle mass measurement (DXA vs BIA) and higher levels of impaired cognition in our population. Sobestiansky et al. [29] reported a mean MMSE score of 28 indicating a particularly good cognitive baseline for the participants compared to the higher percentage of cognitive impairment found in the day hospital group in this study.

The prevalence of probable sarcopenia (reduced muscle strength only) was 23\% using grip strength and 33\% using LL strength. This prevalence was much higher than Dodds et al. [29], when using the EWGSOP2 criteria and grip strength. Dodds et al. [30] established a prevalence of probable sarcopenia of only $5.3 \%$ in the UK Biobank dataset $(N=499,096)$. Of those with probable sarcopenia, only 
Table 3 Associated factors for sarcopenia (grip) and sarcopenia (LL)

\begin{tabular}{|c|c|c|c|c|c|c|c|c|}
\hline \multicolumn{5}{|l|}{ Sarcopenia (grip) } & \multicolumn{4}{|c|}{ Sarcopenia (LL) } \\
\hline Variable & $T$ test & Mann Whitney $U$ & Chi square & Linear-by-linear & $T$ test & Mann Whitney $U$ & Chi square & Linear-by-linear \\
\hline Age & $0.0001 *$ & - & $0.0001 *$ & - & $0.0001 *$ & - & $0.001 *$ & \\
\hline $\mathrm{Hb}$ & - & $0.004 *$ & - & - & - & $0.009 *$ & - & - \\
\hline Height & - & $0.0001^{*}$ & - & - & - & $0.004^{*}$ & - & - \\
\hline Weight & - & $0.0001 *$ & - & - & - & $0.0001 *$ & - & - \\
\hline BMI & - & $0.0001 *$ & - & $0.0001^{*}$ & - & $0.0001 *$ & - & $0.0001 *$ \\
\hline $\mathrm{CC}$ & - & $0.0001 *$ & - & - & - & $0.0001 *$ & - & - \\
\hline Best grip & - & $0.0001 *$ & - & - & - & $0.0001 *$ & - & - \\
\hline Scaf ASM & - & $0.0001 *$ & - & - & - & $0.0001 *$ & - & - \\
\hline Scaf ASM/H & $0.0001^{*}$ & - & - & - & $0.0001 *$ & - & - & - \\
\hline Sergi ASM & - & $0.0001 *$ & - & - & - & $0.0001^{*}$ & - & - \\
\hline Sergi ASM/H & - & $0.0001 *$ & - & - & - & $0.0001 *$ & - & - \\
\hline Gender & - & - & $0.008 *$ & - & - & - & $0.004 *$ & - \\
\hline $\begin{array}{l}\text { Osteoporosis/osteo- } \\
\text { penia }\end{array}$ & - & - & $0.009 *$ & - & - & - & $0.009 *$ & - \\
\hline $\begin{array}{l}\text { LL strength (normal or } \\
\text { reduced) }\end{array}$ & - & - & $0.014 *$ & - & - & - & $0.0001^{*}$ & - \\
\hline $\begin{array}{l}\text { SPPB (normal or } \\
\text { reduced) }\end{array}$ & - & - & 0.015 & - & - & - & $0.001 *$ & - \\
\hline SFI & - & - & $0.04 *$ & $0.02 *$ & - & - & $0.03^{*}$ & $0.005^{*}$ \\
\hline Gait speed 1 & - & $0.046^{*}$ & - & - & - & $0.009 *$ & - & - \\
\hline 5STS time & - & $0.001 *$ & - & - & - & $0.0001^{*}$ & - & - \\
\hline CFS & - & $0.02 *$ & - & $0.023^{*}$ & - & $0.036^{*}$ & - & - \\
\hline MNA-SF & - & $0.049^{*}$ & - & $0.023 *$ & - & $0.026^{*}$ & - & $0.003 *$ \\
\hline
\end{tabular}

$\mathrm{Hb}$, haemoglobin; osteoporosis/osteopenia, history of osteoporosis or osteopenia; BMI, body mass index; CC, calf circumference; scaf ASM, scafoglieri appendicular skeletal mass; scaf ASM/H, scafoglieri appendicular skeletal mass adjusted by height; sergi ASM, sergi appendicular skeletal mass; sergi ASM/H, sergi appendicular skeletal mass adjusted by height; best grip, grip strength (highest score); LL strength (reduced or normal) , 5-STS $>15 \mathrm{~s}$; SPPB (reduced or normal), SPPB $<8$; gait speed 1, $1^{\text {ST }}$ gait speed trail; SFI, shared frailty instrument; CFS, clinical frailty scale; MNA-SF, modified Mini Nutritional Assessment short form

- Not applicable test for variable types

$* p<0.05$

Table 4 Multivariate binary logistic regression model for sarcopenia (grip)

\begin{tabular}{|c|c|c|c|c|c|c|}
\hline & \multirow[t]{2}{*}{$B$} & \multirow[t]{2}{*}{ SE } & \multirow[t]{2}{*}{$p$} & \multirow[t]{2}{*}{ OR } & \multicolumn{2}{|c|}{$95 \% \mathrm{CI}$ for OR } \\
\hline & & & & & Lower & Upper \\
\hline Age & 0.16 & 0.05 & $0.001 *$ & 1.18 & 1.07 & 1.29 \\
\hline Gender & 1.30 & 0.63 & $0.04 *$ & 3.67 & 1.06 & 12.73 \\
\hline $\mathrm{Hb}$ & -0.22 & 0.18 & 0.24 & 0.81 & 0.56 & 1.15 \\
\hline Current_VitD & -0.46 & 0.53 & 0.39 & 0.64 & 0.22 & 1.80 \\
\hline SPPB_Total & 0.04 & 0.11 & 0.72 & 1.04 & 0.84 & 1.30 \\
\hline MNASF_Total & -0.14 & 0.09 & 0.11 & 0.87 & 0.73 & 1.03 \\
\hline CFS & 0.16 & 0.23 & 0.50 & 1.17 & 0.74 & 1.85 \\
\hline CKD & 1.36 & 0.64 & $0.034 *$ & 3.88 & 1.10 & 13.61 \\
\hline Osteopenia osteoporosis & 0.81 & 0.53 & 0.13 & 2.24 & 0.80 & 6.33 \\
\hline
\end{tabular}

age, gender, $H b$ haemoglobin levels, Current_VitD taking vitamin D supplement (yes or no), SPPB_Total short physical performance battery score, $M N A S F_{\_}$Total modified mini nutritional assessment short form total score, CFS CFS category, CKD history of chronic kidney disease, osteopenia osteoporosis history of osteopenia or osteoporosis, $O R$ odds ratio, $C I$ confidence interval, $p$ significance level, $B$ unstandardized coefficients, $S E$ standard error

$* p<0.05$ 
Table 5 Multivariate binary logistic regression model for sarcopenia (LL)

\begin{tabular}{|c|c|c|c|c|c|c|}
\hline & \multirow[t]{2}{*}{$B$} & \multirow[t]{2}{*}{ SE } & \multirow[t]{2}{*}{$p$} & \multirow[t]{2}{*}{ OR } & \multicolumn{2}{|c|}{$95 \% \mathrm{CI}$ for OR } \\
\hline & & & & & Lower & Upper \\
\hline Age & 0.10 & 0.04 & $0.004 *$ & 1.11 & 1.03 & 1.19 \\
\hline Gender & 0.24 & 0.62 & 0.70 & 1.27 & 0.38 & 4.31 \\
\hline Best_Grip & -0.07 & 0.04 & 0.11 & 0.93 & 0.86 & 1.02 \\
\hline MNA_SF_Binary & 1.07 & 0.48 & $0.024 *$ & 2.92 & 1.15 & 7.42 \\
\hline CFS & 0.01 & 0.18 & 0.96 & 1.01 & 0.71 & 1.43 \\
\hline QMCI_Binary & 0.73 & 0.49 & 0.14 & 2.07 & 0.79 & 5.44 \\
\hline $\mathrm{Hb}$ & -0.18 & 0.15 & 0.22 & 0.84 & 0.63 & 1.12 \\
\hline
\end{tabular}

A variable(s) entered on step 1: age, gender, Best_Grip=best grip strength measurement, MNA_SF_ Binary $=$ modified Mini Nutritional Assessment short form (normal or reduced), CFS $=$ Clinical Frailty Scale 1-7, QMCI_Binary=Quick Mild Cognitive Impairment Screen (normal or reduced), Hb=haemoglobin levels. $\mathrm{OR}=$ odds ratio, $\mathrm{CI}=$ confidence Interval, $p=$ significance level, $B=$ unstandardized coefficients, $\mathrm{SE}=$ standard error

$* p<0.05$

$6.3 \%$ of this group had confirmed sarcopenia $(N=1673)$, which represents $0.3 \%$ in the entire data set. The low prevalence of probable sarcopenia may be attributed to the lack of LL strength assessment, low levels of comorbidities and low rate of falls. Of note, across the whole Biobank dataset, $79.9 \%$ had no reported falls $(N=398,801)$ and only $21.4 \%$ $(N=106,507)$ had $>3$ co-morbidities [30].

In our study, the female gender was found to be associated with sarcopenia (Grip) and sarcopenia (LL) as well as being an independently associated factor of sarcopenia (grip). There have been inconsistent findings regarding the relationship between gender and sarcopenia [10, 31, 32]. It is important to note that the differences in how sarcopenia is diagnosed and various associated factors (PA, nutrition, etc.) may influence the relationship between gender and sarcopenia. For example, in Kim and Wong [33], the 5-STS test identified more women as sarcopenic, which may have been influenced by the high prevalence of OA in the female cohort compared to men. The presence of OA has been shown to weaken flexor and extensor muscles of the lower limbs [33]. In comparison to men, women had significantly lower anthropometric measures (height, weight, BMI and CC), increased prevalence of osteoporosis/osteopenia, reduced grip strength, lower m-CIRS score, reduced nutritional state and increased sitting times in this study $(p<0.05)$. The association between the female gender and sarcopenia may be due to their lower BMI and the inability of BIA to distinguish between intra-muscular fat and muscle itself in those with higher BMI (i.e. men).

The muscle mass index (ASM/height) may also have been limited in identifying men as sarcopenic as many would likely be defined as sarcopenic obesity given the increased BMIs. Adjusting muscle mass by BMI has enabled the FNIH criteria to identify those with slower gait speed, increased functional disability and higher BMI [34,
35]. In Newman et al. [36], using the Health ABC study participants $(N=2984)$ the adjustment of ASM by height (using DXA) identified $9 \%$ of males and $0 \%$ of women as sarcopenic with a BMI $>25$. When adjusting ASM by fat mass, sarcopenia prevalence increased to $15-22 \%$ in men and $12-21 \%$ in women for the overweight and obese groups [36]. In Newman et al. [36], sarcopenia was only associated with LL function when defined with ASM adjusted by height and body fat but not height. Further research is warranted to determine the most accurate method to diagnose sarcopenia (height or BMI) adjusted; numerous studies have attempted to address this issue, but more large-scale studies are warranted, especially in European populations.

The most interesting finding from our study was that both types of sarcopenia, defined by grip strength and LL strength, produced different associations and independently associated factors. In previous research, grip strength has been associated with increased risk of mortality, decrease in independence, LL strength, TUG, SPPB and falls [37, 38]. In a similar older adult population, Yeung et al. [39] and McGrath [40], LL strength (knee extension) was found to have a higher association with health characteristics in comparison to handgrip strength while both strength measures were associated with a falls history, reduced gait speed, higher ADL dependence, depression and nutrition. In our study, grip strength was not strongly associated with any of the physical performance measure or 5-STS $(R<0.6)$. Grip strength was only moderately associated with all physical performance measurement and LL strength $(R=0.3-0.59)$.

Grip strength has been recommended as a surrogate measure of LL strength as it is highly correlated and grip strength and easier to measure in clinical practice [41]. Grip strength and LL strength have shared similar associations with physical performance measures in community-dwelling populations, but the strength of the association can vary 
[39]. Fragala et al. [42] identified both LL strength (isokinetic) and grip strength as predictors of slow gait, with LL strength a slightly stronger association compared to grip strength in two large datasets. Fragala et al. [42] found that LL strength was a better predictor of gait speed, compared to grip strength when a faster gait speed was reported (higher functioning). This indicates that the association between grip strength and gait speed was less when the population had a higher gait speed/performed at a higher function. In addition, Fragala et al. [42] highlighted that the relationship between grip strength and gait speed may be more attenuated in those with severe weakness. This finding has implications for clinical practice as it depicts that grip strength is not a suitable strength measurement for higher functioning older adults when predicting a relationship to adverse to functional outcomes. It may be that grip strength may be more suitable assessment for older and less functional patients, where those younger and more functional will require a LL strength test.

Acknowledging the application of both grip strength and LL strength testing in a different population, Manini and Clark [43] developed an assessment algorithm for dynapenia (reduced muscle strength). In this algorithm, the use of grip strength was used to determine the need to assess LL strength. For example, if a person recorded a low grip strength measure, a LL test must be complete, if grip strength was normal, no further strength tests were required to be carried out. The authors argue the inclusion of both grip strength and LL strength, to be included, to out rule a cause of muscle strength being neurological vs. muscular in origin [43]. This process also ensures those with specific issues affecting grip strength or LL strength are not incorrectly classified as dynapenic (weak), considering grip strength is only a surrogate measure of overall strength [43]. Therefore, the inclusion of grip strength and LL strength may enable greater precision in the identification of those at risk of adverse outcomes and aid sarcopenia identification [38].

Ours is the first study to apply the EWGSOP2 criteria to older adults living in the community in an Irish day hospital setting. The findings highlight the feasibility of applying the EWGSOP2 screening process easily and safely in this setting. Bias was minimised as much as possible, using a robust methodology and the use of standardised assessments and instructions. The study protocol was designed, with a key focus on reproducibility and transparency to enable future research to implement similar designs, to compare findings accurately.

Due to cross-sectional design of the study, no predictive factors of sarcopenia could be identified, only associations could be determined between sarcopenia and other factors.
Another limitation was the failure to reach the planned sample size of 180 . The final sample size of the study was 134 and this was due to the need to cease clinical research activity in the RMDH due to the COVID-19 pandemic in early March 2020. There are several weaknesses associated with the assessment procedure adopted. For BIA analysis, participants did not follow a strict fluid restriction for $24 \mathrm{~h}$ prior to assessment or void bladder immediately prior. Given that BIA is influenced by fluid status, the lack of standardised procedure may have limited the accuracy of findings.

In conclusion, the method of strength testing influenced which variables were independently associated with sarcopenia, potentially highlighting different pathophysiological changes between the two types of diagnosis for sarcopenia. To appropriately manage sarcopenia, a greater understanding is required. Firstly, the assessment of sarcopenia needs be more consistent regarding the use of the EWGSOP2 guidelines, the assessment of muscle strength (grip or 5-STS or both) and muscle mass assessment (BIA, DXA, MRI or perhaps ultrasound). In relation to muscle mass assessment, further research is required to determine the most appropriate muscle mass index. The use of other assessment tools (e.g. ultrasound) in the clinical setting needs to be considered and validated against gold standards of muscle mass, such as MRI or CT. Routine assessment of sarcopenia would enable a greater understanding of the factors that influence it (i.e. falls or hospitalisation) and the changes in sarcopenia after hospitalisation or acute illness. In the meantime, the results of the present study underscore the clinical relevance of the assessment of sarcopenia in the geriatric day hospital setting, and it is recommended that beyond research studies, the routine clinical assessment of sarcopenia in this setting is incorporated as part of existing and future healthcare policies to improve outcomes from potentially remediable conditions in the older population.

Acknowledgements We wish to thank the study gatekeepers and all participants who took part in the study. The study was funded by an Irish Research Council Employment-Based Postgraduate Program Award (EBPPG/2019/50) in association with St James's Hospital, Dublin, Ireland.

Author contributions $\mathrm{KC}$ completed the research as part of a research. $\mathrm{MSc}$ Prof FH and Prof RR-O were involved in the development of the research design, data collection and data analysis. Prof RR-O assisted with data analysis. All authors contributed to the design of the study, reviewed, and approved the manuscript.

Funding This work was supported by the Irish Research Council through an Employment-Based Postgraduate Scholarship (EBPPG/2019/50).

Availability of data and material All data or material will be available upon request to the main corresponding author. 


\section{Compliance with ethical standards}

Conflict of interest No conflict of interest to declare.

Ethics approval Ethical approval was granted by the Research Ethics Committee of SJH and Tallaght University Hospital (2019-10 List 39 [09]) in October 2019. The research study was also approved by the St James's Hospital Research and Innovation department (Reference number: 5672).

Consent to participate Informed Consent (verbal and written) was obtained from all individual participants included in the study.

Consent for publication Participants signed informed consent regarding publishing their data (non-identifiable).

\section{References}

1. Marzetti E, Calvani R, Tosato M, Cesari M, Bari M, Cherubini A, Collamati A, D’Angelo E, Pahor M, Bernabei R, Landi F (2017) Sarcopenia: an overview. Aging Clin Exp Res 29(1):11-17

2. Sayer A, Robinson S, Patel H, Shavlakadze T, Cooper C, Grounds M (2013) New horizons in the pathogenesis, diagnosis and management of sarcopenia. Age Ageing 42(2):145-150

3. Witham $M(2019)$ Bridging the gap between the laboratory and the clinic for patients with sarcopenia. Biogerontology 20(2):241-248

4. Solane P, Marzetti E, Lindi F, Zimmerman S (2019) Understanding and addressing muscle strength, mass, and function in older persons. JAMDA 20(1):1-4

5. Nascimento P, Poitras S, Bilodeau M (2018) How do we define and measure sarcopenia? Protocol for a systematic review. Syst Rev 7:51-60

6. Cruz-Jentoft A, Bahat G, Bauer J, Boirie $\mathrm{Y}$, Bruyère $\mathrm{O}$, Cederholm T, Cooper C, Landi F, Rolland Y, Sayer A, Schneider S, Sieber C, Topinkova E, Vandewoude M, Visser M, Zamboni M, Bautmans I, Baeyens J, Cesari M, Cherubini A, Kanis J, Maggio M, Martin F, Michel J, Pitkala K, Reginster J, Rizzoli R, SánchezRodríguez D, Schols J (2019) Sarcopenia: revised European consensus on definition and diagnosis. Age Ageing 48(1):16-31

7. Dos Santos L, Cyrino ES, Antunes M, Santos DA, Sardinha LB (2017) Sarcopenia and physical independence in older adults: the independent and synergic role of muscle mass and muscle function. J Cachexia Sarcopenia Muscle 8(2):245-250

8. Central Statistics Office (CSO) (2016) Population and Labour Force Projections 2017-2051. Dublin: Central Statistics Office, www.CSO.ie. https://www.cso.ie/en/releasesandpublications/ ep/p-plfp/populationandlabourforceprojections2017-2051/popul ationprojectionsresults/. Accessed 1 Aug 2019

9. Elliot JA, Doyle SL, Murphy CF, King S, Guinan EM, Beddy P, Ravi N, Reynolds JV (2017) Sarcopenia: prevalence, and impact on operative and oncologic outcomes in the multimodal management of locally advanced esophageal cancer. Ann Surg 266(5):822-830

10. Cruz-Jentoft A, Baeyens J, Bauer J, Boire Y, Cederholm T, Landi F, Martin F, Michel J, Rolland Y, Schneider S, Topinkov E, Vandewoude M, Zamboni M (2010) Sarcopenia: European consensus on definition and diagnosis. Age Ageing 39:412-423

11. National Clinical Programme for Older People (2012) Specialist Geriatric Services Model of Care'. Health Service ExecutiveNational Clinical Programme for Older people committee. https:// www.hse.ie/eng/services/publications/clinical-strategy-and-progr ammes/specialist-geriatric-services-model-of-care.pdf. Accessed 5 Feb 2020
12. O'Caoimh R, Kennelly S, O'Shea D (2018) Can the geriatric day hospital act as a hub for services for older people across the spectrum of ageing from active ageing to advanced frailty? Front Med 5(23):1-4

13. Parmelee PA, Thuras PD, Katz IR, Lawton MP (1995) Validation of the cumulative illness rating scale in a geriatric residential population. J Am Geriatr Soc 43(2):130-137

14. Salvi F, Miller M, Grilli A, Giorgi R, Towers A, Morichi V, Spazzafumo L, Mancinelli L, Espinosa E, Rappelli A, Dessi-Fulgheri P (2008) A Manual of guidelines to score the modified cumulative illness rating scale and its validation in acute hospitalized elderly patients. J Am Geriatr Soc 56(10):1926-1931

15. Roberts H, Denison H, Martin H, Patel H, Syddall H, Cooper C, Sayer A (2011) A review of the measurement of grip strength in clinical and epidemiological studies: towards a standardised approach. Age Ageing 40(4):423-429

16. Guralnik J, Simonsick E, Ferrucci L, Glynn R, Berkman L, Blaer D, Scherr P, Wallace R (1994) A short physical performance battery assessing lower extremity function: association with selfreported disability and prediction of mortality and nursing home admission. J Gerontol Med Sci 49(2):85-94

17. Sergi G, De Rui M, Veronese N, Bolzetta F, Berton L, Carraro S, Bano G, Coin A, Manzato E, Perissinotto E (2015) Assessing appendicular skeletal muscle mass with bioelectrical impedance analysis in free-living Caucasian older adults. Clin Nutr 34(4):667-673

18. Scafoglieri A, Clarys JP, Bauer J, Verlaan S, Van Malderen L, Vantieghem S, Cederholm T, Sieber C, Mets T, Bautmans I (2017) Predicting appendicular lean and fat mass with bioelectrical impedance analysis in older adults with physical function decline the PROVIDE study. Clin Nutr 36(3):869-875

19. Herman T, Giladi N, Hausdorff JM (2011) Properties of the 'timed up and go' test: more than meets the eye. Gerontology 57(3):203-210

20. Kaiser M, Uter W, Bauer J, Guigoz Y (2009) Validation of the Mini Nutritional Assessment Short-Form (MNA(r)-SF) a practical tool for identification of nutritional status. J Nutr Health Aging 13(9):782-790

21. Glynn, k., Coen, R. \& Lawlor, B. (2019) Is the Quick Mild Cognitive Impairment Screen (QMCI) more accurate at detecting mild cognitive impairment than existing short cognitive screening tests? A systematic review of the current literature. Int J Geriatr Psychiatry 34(12):1739-1746

22. Romero-Ortuno R, O'Shea D, Kenny RA (2011) The SHARE Frailty Instrument for primary care predicts incident disability in a European population-based sample. Prim Care 19(5):301-309

23. Sjóstróm M, Ainsworth BE, Bauman A, Bull F, Hamilton-Craig CR, Sallis JF (2005) Guidelines for data processing analysis of the International Physical Activity Questionnaire (IPAQ) — short and long forms. https://sites.google.om/site/theipaq/scoring-protocol. Accessed Aug 2019

24. Conroy R (2016) The RCSI sample size handbook. Royal College of Surgeons in Ireland, Dublin. https://www.semanticscholar .org/paper/The-RCSI-Sample-size-handbook-Conroy/86adada0c0 d7191f5eda28b2966d7ced648fcab3. Accessed Aug 2019

25. Peduzzi P, Concato J, Kemper E, Holford TR, Feinstein AR (1996) A simulation study of the number of events per variable in logistic regression analysis. J Clin Epidemiol 49(12):1373-1379

26. Dodds M, Granic A, Davies K, Kirkwood T, Jagger C, Sayer A (2017) Prevalence and incidence of sarcopenia in the very old: findings from the Newcastle 85+ Study. J Cachexia, Sarcopenia Muscle 8(2):229-237

27. Cuesta F, Formiga F, Lopez-soto A, Masanes F, Ruiz D, Artaza I, Salva A, Serra-Rexach J, Luque X, Crux-Jentoft A (2015) Prevalence of sarcopenia in patients attending outpatient geriatric clinics: the ELLI study. Age Ageing 44:807-809 
28. Reiss J, Alzner R, Mayr-Pirker B, Pirich C, Kassmann H, Kreutzer M, Dovjak P, Reiter R (2019) Consequences of applying the new EWGSOP2 guideline instead of the former EWGSOP guideline for sarcopenia case finding in older patients. Age Ageing 48(5):713-718

29. Sobestiansky S, Michaelsson K, Cederholm T (2019) Sarcopenia prevalence and associations with mortality and hospitalisation by various sarcopenia definitions in 85-89 year old communitydwelling men: a report from the ULSAM study. BMC Geriatr 19(1):318

30. Dodds R, Granic A, Robinson S, Sayer A (2019) Sarcopenia, longterm conditions, and multimorbidity: findings from UK Biobank participants. J Cachexia, Sarcopenia Muscle 11(1):62-68

31. Shafiee G, Keshtkar A, Soltnai A, Ahadi Z, Larijani B, Heshmat R (2017) Prevalence of sarcopenia in the world: a systematic review and meta-analysis of general population studies. J Diabetes Metab Disord 16:21-31

32. Kirchengast S, Huber J (2009) Gender and age differences in lean soft tissue mass and sarcopenia among healthy elderly. Anthropologischer Anzeiger; Bericht uber die biologisch-anthropologische Literature 67(2):139-151

33. Kim M, Won C-W (2019) Prevalence of sarcopenia in communitydwelling older adults using the definition of the European working group on sarcopenia in older people 2: findings from the Korean frailty and aging cohort study. Age Ageing 48(6):910-916

34. Dam T-T, Peters K, Fragala M, Cawthon P, Harris T, McLean R, Shardell M, Alley D, Kenny A, Ferrucci L, Guralnik J, Kiel D, Kritchevsky S, Vassileva M, Studenski S (2014) An evidencebased comparison of operational criteria for the presence of sarcopenia. J Gerontol A Biol Sci Med Sci 69(5):584-590

35. Tessier A-J, Wing S, Rahme E, Morais J, Chevalier S (2019) Physical function-derived cut-points for the diagnosis of sarcopenia and dynapenia from the Canadian longitudinal study on aging. $\mathbf{J}$ Cachexia Sarcopenia Muscle 10(5):985-999

36. Newman A, Kupelian V, Visser M, Simonsick E, Goodpaster B, Nevitt M, Kritchevsky S, Tylavsky F, Rubin S, Harris T (2003)
Sarcopenia: alternative definitions and associations with lower extremity function. J Am Geriatr Soc 51(11):1602-1609

37. Beaudart C, Rolland Y, Cruz-Jentoft AJ, Bauer JM, Sieber C, Cooper C, Al-Daghri N, Araujo de Carvalho I, Bautmans I, Bernabei $\mathrm{R}$, Bruyère $\mathrm{O}$, Cesari $\mathrm{M}$, Cherubini $\mathrm{A}$, Dawson-Hughes B, Kanis JA, Kaufman JM, Landi F, Maggi S, McCloskey E, Petermans J, Fielding RA (2019) Assessment of muscle function and physical performance in daily clinical practice : a position paper endorsed by the european society for clinical and economic aspects of osteoporosis, osteoarthritis and musculoskeletal diseases (ESCEO). Calcif Tissue Int 105(1):1-14

38. McGrath R, Johnson N, Klawitter L, Mahoney S, Trautman K, Carlson C, Rockstad E, Hackney KJ (2020) What are the association patterns between handgrip strength and adverse health conditions? A topical review. SAGE Open Med 8:2050312120910358

39. Yeung SS, Reijnierse EM, Trappenburg MC, Blauw GJ, Meskers CG, Maier AB (2018) Knee extension strength measurements should be considered as part of the comprehensive geriatric assessment. BMC Geriatr 18(130): 1-8

40. McGrath R (2019) Understanding the feasibility and validity of muscle strength measurements in aging adults. JAMDA 20(1):99-100

41. Bohannon RW (2019) Grip strength: an indispensable biomarker for older adults. Clin Interv Aging 14:1681-1691

42. Fragala MS, Alley DE, Shardell MD, Harris TB, McLean RR, Kiel DP, Cawthon PM, Dam TT, Ferrucci L, Guralnik JM, Kritchevsky SB, Vassileva MT, Gudnason V, Eiriksdottir G, Koster A, Newman A, Siggeirsdottir K, Satterfield S, Studenski SA, Kenny AM (2016) Comparison of handgrip and leg extension strength in predicting slow gait speed in older adults. J Am Geriatr Soc 64(1):144-150

43. Manini TM, Clark BC (2012) Dynapenia and aging: an update. J Gerontol Ser A Biol Sci Med Sci 67(1):28-40

Publisher's Note Springer Nature remains neutral with regard to jurisdictional claims in published maps and institutional affiliations. 